\title{
Performance Comparison of Wavelet Transform and Contourlet Transform based methods for Despeckling Medical Ultrasound Images
}

\author{
P.S. Hiremath \\ Dept. of P.G. Studies and \\ Research in Computer Science \\ Gulbarga University, Gulbarga- \\ 585106 , \\ Karnataka, India.
}

\author{
Prema T. Akkasaligar \\ Dept. of Computer Science \& \\ Engineering, B.L.D.E.A's Dr \\ P.G.H. Engineering College, \\ Bijapur-586103, Karnataka, \\ India.
}

\author{
Sharan Badiger \\ Sri.B.M.Patil Medical College, \\ BLDE University, Bijapur- \\ 586103, \\ Karnataka, India.
}

\begin{abstract}
The widespread usage of ultrasound imaging equipment necessitates the need for better image processing techniques to offer a clearer image to the medical practitioner. This makes the use of efficient despeckle filtering a very important task. The speckle is most often considered a dominant source of multiplicative noise in ultrasound imaging and should be filtered out without affecting important features of the image. The objective of the paper is to compare performance of the multiscale methods, namely, wavelet transform, Laplacian pyramid transform and contourlet transform for despeckling medical ultrasound images. The effects of different thresholding techniques using Bayes shrinkage rule for denoising ultrasound images are examined. The despeckled image quality is evaluated using filter assessment parameters like variance, Mean-Square Error (MSE), Signal to Noise Ratio (SNR), Peak Signal to Noise Ratio (PSNR) and Correlation Coefficient (CC). The performance comparison of multi-scale schemes shows that contourlet transform based despeckling method excels over the other two transform based despeckling methods. Extensive experimentation has been carried out for comparative analysis of performance of the methods.
\end{abstract}

\section{General Terms}

Image analysis, Noise reduction.

\section{Keywords}

Despeckling, Wavelet transform, Contourlet transform, Laplacian pyramid transform.

\section{INTRODUCTION}

Medical images are usually corrupted by noise during their acquisition and transmission. The main objective of image denoising techniques is to remove such noises while retaining as much as possible the important image features. The use of ultrasound (US) in diagnosis is well established because of its non-invasive nature, low cost, capability of forming real time imaging and continuing improvement in image quality [1]. However, the usefulness of ultrasound imaging is degraded by the presence of signal dependant noise known as speckle. So speckle filtering is a central pre-processing step for feature extraction, analysis and recognition from medical imagery measurements.

The post acquisition techniques for speckle reduction can be classified into two categories: single scale spatial filtering and transform domain filtering methods. Within the second category, several multiscale methods have been proposed. Recently, there have been many challenges to reduce the speckle noise using Wavelet Transform (WT) as a multiresolution image processing tool. Speckle noise is a high-frequency component of the image and appears in wavelet coefficients. One widespread method used for speckle reduction is wavelet shrinkage. In [2], a review of despeckling methods for ultrasound imaging is presented. N.K.Ragesh et al. [3] have discussed about two broad categories of noise reduction techniques in ultrasound images namely during acquisition and after acquisition of image. Hiremath et al. [4] have proposed despeckling ultrasound images using wavelet and Bayes thresholding. Alin Achim et al. [5] have presented a novel speckle suppression method for medical ultrasound images. The authors design a Bayesian estimator that exploits speckle statistics. Aleksandra Pizurica et al. [6] have proposed a robust wavelet domain method for noise filtering in medical images. Nikhil Gupta and M.N. Swamy [7] have proposed a novel technique for despeckling the medical ultrasound images using lossy compression and wavelet transform. There has been growing awareness to the observation that wavelet transform is not capable of diagnosing the direction of any line-shaped discontinuity in the image [6]. In essence, wavelets are good at catching zero dimensional singularities, but two-dimensional piecewise smooth signals resembling images have onedimensional singularities. That is, smooth regions are separated by edges, and while edges are discontinuous across, they are typically smooth curves. Intuitively, wavelets in 2-D obtained by a tensor-product of one dimensional wavelets will be good at isolating the discontinuity across the edge, but will not see the smoothness along the edge. This disappointing behavior indicates that more powerful bases are needed in higher dimensions.

A new nonseparable two-dimensional signal transform, called the Contourlet Transform (CT) has recently been proposed as an alternative to an improvement on separable wavelet for representation of natural images. This transform scales to capture the intrinsic geometrical structure in visual information through a multiresolution, multidimensional decomposition [8]. Mao-Yu Huang et al. [9] have presented a contourlet based speckle reduction method for breast ultrasound images. The double iterated filter bank structure and a small redundancy at most $4 / 3$ using two thresholding methods shows a great promise for speckle reduction. Hiremath et al. [10] have proposed 
despeckling medical ultrasound images using contourlet transform using Bayes shrinkage rule. K. Thangavel et al. [11] have compared different filtering techniques based on statistical methods for the removal of speckle noise from ultrasound image of prostate. Wan M.H. et al. [12] have compared performance of five common enhancement techniques based on spatial domain filtering, frequency domain filtering, histogram processing, morphological filtering and wavelet filtering for ultrasound kidney image. Authors show that detection of edge is better by using wavelet filtering and median filtering. Mariana et al. [13] have compared despeckling method, based on wavelet transform, the median filter and the wiener filter for medical ultrasound images. By means of experimental results, it has been shown that the WT method yields far better results than the other two filters. Ali Saad [14] has compared two multi resolution methods: WT and Laplacian pyramid transform (LP), for simultaneous speckle reduction and contrast enhancement for ultrasound images. As a lot of variability exists in ultrasound images, the wavelet method proves to be a much better method than the Laplacian one for an overall improvement. However, a comparison of all the three transforms, namely wavelet transform, Laplacian pyramid transform, and contourlet transform is not found in the literature. In this paper, the aim is to study comparative analysis of the above multiresolution methods for despeckling medical ultrasound images.

\section{THE TRANSFORM BASED DESPECK- -LING ALGORITHM}

The noise commonly manifests itself as a fine grained structure in an image, which leads to discontinuities at edge points. The transform exploits smoothness of contour effectively by considering a variety of directions following the contours. Further, thresholding is performed so as to reduce speckle. The steps involved in the proposed method are given in the Algorithm 1.

Algorithm 1: Despeckling of ultrasound image.

Input: Medical ultrasound image.

Output: Despeckled image.

Start

Step 1: Input medical ultrasound image X.

Step 2: Apply log transformation to the input image X.

Step 3: Apply the wavelet transform on the log transformed image of the Step 2 upto $n$ levels of subband decomposition at each level.

Step 4: Perform thresholding of the transformed image of the Step 3.

Step 5: By performing the inverse transform on the thresholded image of the Step 4, the despeckled image Y is obtained (output image).

Step 6: Compute the values of the performance parameters, namely, variance, MSE, SNR,PSNR, correlation coefficient for the despeckled image Y of the Step 5.

Stop

In the Step 4, the wavelet transformed image can be thresholded by : (i) selecting either global or subband thresholding function, (ii) selecting shrinkage scheme (Hard, Soft or Semi-soft), and (iii) selecting Bayes shrinkage or universal shrinkage rule. However, in the Step 3, the Laplacian pyramidal transform or the contourlet transform can be employed instead of the wavelet transform. The LP transform is performed using different filters upto $\mathrm{n}$ levels, where $\mathrm{n}$ depends on the image size. The contourlet transform can be done upto $\mathrm{n}$ levels of Laplacian pyramidal decomposition and $\mathrm{m}$ directional decompositions at each level, where $\mathrm{n}$ and $\mathrm{m}$ depend on the image size. This is followed by the thresholding in the Step 4 by selecting shrinkage scheme (Hard, Soft or Semi-soft), and using Bayes shrinkage rule.

\section{TRANSFORM METHODS}

The transform methods are based on the discrete wavelet transform, the Laplacian pyramid transform and the contourlet transform, which are described below:

\subsection{Discrete wavelet transform}

Wavelets have recently emerged as a powerful tool for image denoising. The discrete wavelet transform maps an image into a set of coefficients that constitute a multiscale representation of the image. Discrete Wavelet Transform (DWT) of a signal $x(n)$ is computed by passing it through a series of filters. First the samples are passed through a low pass filter with impulse response $g(n)$ giving approximation coefficients. The signal is decomposed simultaneously using a high pass filter $h(n)$, giving the detailed coefficients. The low pass filter gives approximation coefficients. These filters are called Quadrature Mirror Filters (QMF). Since half the frequencies of the signal are removed, the filter outputs are down sampled by 2 .

$$
\begin{array}{ll}
Y_{\text {low }}[k]=\sum_{n} x[n] \cdot g[2 k-n] & \text { low pass filter output. } \\
Y_{\text {high }}[k]=\sum_{n} x[n] \cdot h[2 k-n] & \text { high pass filter output. }
\end{array}
$$

Image is a $2 \mathrm{D}$ signal represented by $\mathrm{x}(\mathrm{N}, \mathrm{M})$. Each row is filtered and then down sampled to obtain two $(\mathrm{N}, \mathrm{M} / 2)$ images. Then each column is filtered and down sampled to obtain four $(\mathrm{N} / 2, \mathrm{M} / 2)$ images. The four subbands created are named as $\mathrm{LL}$, $\mathrm{LH}, \mathrm{HL}$ and $\mathrm{HH}$. In two dimensions, following functions are required: (i) One two-dimensional scaling function $\varphi(x, y)$. (ii)Three two-dimensional wavelet functions, $\psi^{H}(x, y), \psi^{V}(x, y)$ and $\psi^{D}(x, y)$. The Approximation subband LL is then decomposed into four at level two and the process can be continued in the same manner for further levels. LL subband has the energy concentration for low pass and the HH band contains high frequency components. Reconstruction can be performed by IDWT (Inverse Discrete Wavelet Transform) to obtain the despeckled image. The despeckling using wavelet coefficients is has been experimented in [4].

\subsection{Laplacian pyramid transform}

One way of achieving a multiscale decomposition is to use a Laplacian pyramid (LP) [15]. The LP decomposition at each level generates a down sampled lowpass version of the original as coarse approximation ' $c$ ' and the difference ' $d$ ' between the original and the prediction, resulting in a band pass image. The process can be iterated by decomposing the coarse version repeatedly. Assuming the filters in LP are orthogonal filters, an image $\mathrm{X}$ is decomposed into $\mathrm{J}$ bandpass images $\mathrm{d}_{\mathrm{j}}$ $, j=1,2, \ldots, J$ and a coarse approximation image $c_{j}$. Then, we have $\|\mathrm{X}\|^{2}=\sum_{j=1}^{J}\left\|d_{j}\right\|^{2}+\left\|c_{J}\right\|^{2}$.

The Laplacian is then computed as the difference between the original image and the low pass filtered image. This process is continued to obtain a set of band-pass filtered images (since each one is the difference between two levels of the Gaussian pyramid). Thus the Laplacian pyramid is a set of band pass filters. By repeating these steps several times, a sequence of images are obtained. If these images are stacked one above 
another, the result is a tapering pyramid data structure and, hence the name the Laplacian pyramid. This can thus be used to represent images as a series of bandpass filtered images, each sampled at successively sparser densities. A drawback of the LP is the implicit oversampling. However, in contrast to the critically sampled wavelet scheme, the LP has the distinguishing feature that each pyramid level generates only one bandpass image (even for multidimensional cases), which does not have "scrambled" frequencies. This frequency scrambling happens in the wavelet filter bank when a highpass channel, after downsampling, is folded back into the low frequency band, and thus its spectrum is reflected. In the LP, this effect is avoided by downsampling the lowpass channel only.

\subsection{Contourlet transform}

An efficient representation of visual information is one of the important tasks in image processing applications such as denoising. Efficiency of a representation refers to the ability to capture significant information of an object of interest using a small description. Medical ultrasound images are not simply stacks of 1-D piecewise smooth scan-lines. The discontinuity points (i.e. edges) are typically located along smooth curves (i.e. contours) owing to smooth boundaries of physical objects. Thus these images contain intrinsic geometrical structures that are key features in visual information. The wavelets in 2-D are good at isolating the discontinuities at edge points, but will not see the smoothness along the contours. The contourlets can be loosely interpreted as a grouping of nearby wavelet coefficients since their locaters are locally correlated due to the smoothness of the boundary curve. We can obtain a sparse image expansion by first applying a multiscale transform and then applying a local directional transform to gather the nearby basis functions at the same scale into linear structures. Thus, we perform a wavelet like transform for edge detection, and then a local directional transform for contour segment detection. In other words, the contourlet transform comprises a Double Filter Bank (DFB) approach for obtaining sparse expansions for typical images with smooth contours. The contourlet transform is the simple directional extension for wavelet that fixes its subband mixing problem and improves its directionality. The continuous-domain expansion generated by the contourlet construction [16] assumes that the Laplacian pyramid is a multiscale decomposition of the $L^{2}\left(R^{2}\right)$ space into a series of increasing resolution

$$
L^{2}\left(R^{2}\right)=V_{j_{0}} \oplus\left(\underset{j=j 0}{\oplus_{j} W_{j}}\right)
$$

with the usual definition of the subspaces $V_{j 0}$ and $W_{j}$ as in the wavelet multiresolution analysis: $V_{j o}$ is an approximation subspace at the scale $2^{\mathrm{j} 0}$, whereas $W_{j}$ contains the added details to the fine scale $2^{\mathrm{j}-1}$. In contourlet transform, suppose that an $1_{\mathrm{j}}$-level DFB is applied to the detail subspace $W_{j}$ of the LP. This results in a decomposition of $W_{j}$ into $2^{1}$ directional subspaces at scale $2^{\mathrm{j}}$ :

$$
W_{j}=\bigoplus_{k=0}^{2_{j}^{l}-1} W_{\mathrm{j}, \mathrm{k}}^{1_{\mathrm{j}}} .
$$

The band pass images $\left(\mathrm{d}_{\mathrm{j}}[\mathrm{n}]\right)$ from the LP are fed into a DFB so that directional information can be captured. The scheme can be iterated on the coarse image $\left(c_{j}[n]\right)$. The combined result is a double iterated filter bank structure, named Pyramidal Directional Filter Bank, (PDFB), which decomposes images into directional subbands at multiple scales. The contourlet expansion satisfies the parabolic scaling relation for curves: width $\alpha$ length ${ }^{2}$ by using directional decompositions, where the number of directions is doubled at every other finer scale. Assuming the DFB is orthogonal, it decomposes each bandpass image $b_{\mathrm{j}}$ into directional coefficients $\mathrm{d}_{\mathrm{j}}$ with $\left\|b_{j}\right\|^{2}=\left\|d_{j}\right\|^{2}$. Then the decomposition by PDFB : $\mathrm{X} \mapsto\left(\mathrm{d}_{1}, d_{2}, \ldots d_{J}, a_{J}\right)$ also has the energy conservation property:

$$
\|X\|^{2}=\sum_{j=1}^{J}\left\|d_{j}\right\|^{2}+\left\|a_{J}\right\|^{2} .
$$

\section{ADAPTIVE THRESHOLDING}

The thresholding approach is sensitive to noise components. In this approach, noisy transform coefficients (high frequency components) are attenuated, whose amplitudes are smaller than a certain statistical threshold value, to zero while retaining the smoother transform coefficients to reconstruct the ideal image without much loss in its details. In Soft Thresholding (ST) technique, the input is shrunk to zero by an amount of threshold $\lambda$. In Hard Thresholding (HT) technique, the input is preserved if it is greater than threshold $\lambda$; otherwise, it is set to zero. The aim of Semi-Soft Threshold (SST) is to offer a compromise between hard and soft thresholding by changing the gradient of the slope. This scheme requires two thresholds, a lower threshold $\lambda$ and an upper threshold $\lambda_{1}$ where $\lambda_{1}$ is estimated to be twice the value of lower threshold $\lambda$. The criterion of each scheme is described as follows. Given that $\lambda$ denotes the threshold limit, $X_{w}$ denotes the input transform coefficients and $\mathrm{Y}_{\mathrm{t}}$ denotes the output transform coefficients after thresholding, we define the following thresholding functions:

(i) Hard Thresholding

$$
\begin{aligned}
\mathrm{Y}_{t} & =\mathrm{T}_{\text {hard }}\left(\mathrm{X}_{w}\right) \\
& =\left\{\begin{array}{lll}
\mathrm{X}_{w}, & \text { where } & \left|\mathrm{X}_{w}\right| \geq \lambda \\
0 \quad, & & \left|\mathrm{X}_{w}\right|<\lambda
\end{array}\right\}
\end{aligned}
$$

(ii) Soft Thresholding

$$
\begin{aligned}
& \mathrm{Y}_{\mathrm{t}}=\mathrm{T}_{\text {soft }}\left(\mathrm{X}_{w}\right)
\end{aligned}
$$

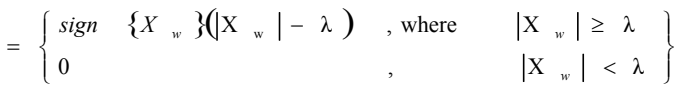

(iii) Semi-soft Thresholding

$$
\begin{aligned}
& \mathrm{Y}_{\mathrm{t}}=\mathrm{T}_{\text {semi }- \text { soft }}\left(\mathrm{x}_{w}\right) \\
& =\left\{\begin{array}{lc}
0 & , \text { where } \quad\left|\mathrm{X}_{\mathrm{w}}\right| \leq \lambda, \\
\operatorname{sign}\left\{\mathrm{X}_{\mathrm{w}} \frac{\lambda_{1}\left(\left|\mathrm{X}_{\mathrm{w}}\right|-\lambda\right)}{\lambda_{1}-\lambda},\right. & \text { where } \lambda<\left|\mathrm{X}_{\mathrm{w}}\right| \leq \lambda_{1} \\
\mathrm{X}_{w} & , \text { where } \quad\left|\mathrm{X}_{\mathrm{w}}\right|>\lambda_{1}
\end{array}\right\} \\
& \text { where } \lambda_{1}=2 \lambda
\end{aligned}
$$

\subsection{Bayes shrinkage rule}

This shrinkage rule uses a Bayesian mathematical frame work for images to derive subband dependent thresholds. The formula for the threshold on a given subband s for the model, with zero mean variable $\mathrm{X}$, is given by

$$
\lambda_{s}=\frac{\sigma_{n}^{2}}{\sigma_{x}},
$$

where $\sigma_{n}$, the estimated noise variance found as the median of the absolute deviation of the transform coefficients on the finest level $L_{l}$, is given by

$$
\sigma_{n}=\frac{\operatorname{median}\left(\left\{\left|\mathrm{X}_{i j}\right| \in L_{1}\right\}\right)}{0.67452}
$$


$\sigma_{x}$, the estimated signal variance on the sub band considered, is given by

$$
\sigma_{x}=\sqrt{\operatorname{Max}\left(\sigma_{y}^{2}-\sigma_{n}^{2}, 0\right)},
$$

and $\sigma_{y}^{2}$, an estimate of the variance of the observations, is given by

$$
\sigma_{y}^{2}=\frac{1}{N_{s}} \sum_{k=1}^{N_{s}} W_{k}^{2}
$$

in which, $\mathrm{N}_{\mathrm{s}}$ is the number of the transform coefficients $\mathrm{W}_{\mathrm{k}}$ on the subband considered. In the Eq. 11, the value 0.67452 is the median absolute deviation of normal distribution with zero mean and unit variance. In case of $\sigma_{n}^{2} \geq \sigma_{y}^{2}, \sigma_{x}$ is zero, and in practice, $\quad \lambda_{s}\left(\sigma_{X}\right)=\max \left\{\left|\mathrm{X}_{\mathrm{i}, \mathrm{j}}\right| \in L_{1}\right\} \quad$ and $\quad$ all coefficients are set to zero.

\section{FILTER ASSESSMENT}

To quantify performance improvements of the speckle reduction method, various measures are used. The following metrics are calculated for the original image $\mathrm{X}$ and the despeckled image $\mathrm{Y}$. Noise Variance: It determines the contents of the speckle in the image. A lower variance gives a cleaner image as more speckles are reduced. The formula for calculating the variance $\sigma$ is

$$
\sigma=\frac{1}{N} \sum_{j=0}^{N-1} \mathrm{X}_{j}^{2}
$$

Mean Square Error (MSE): The MSE measures the quality change between the original image $(\mathrm{X})$ and denoised image $(\mathrm{Y})$. The average of the squared error measure is given by

$$
\mathrm{MSE}=\frac{1}{N} \sum_{j=0}^{N-1}\left(\mathrm{Y}_{\mathrm{j}}-\mathrm{X}_{j}\right)^{2} .
$$

The MSE has been widely used to quantify image quality and, when used alone, it does not correlate strongly enough with perceptual quality. It should be used, therefore, together with other quality metrics and visual perception.

Signal-to-Noise Ratio (SNR): The SNR represents the size of the error relative to the signal. We can find the ratio of the average squared value of the source and the MSE as SNR. It is expressed in decibels $(\mathrm{dB})$ as

$$
\mathrm{SNR}=10 \log _{10}\left(\frac{\sigma_{g}{ }^{2}}{\sigma_{e}^{2}}\right),
$$

where, $\sigma_{g}^{2}$ is the variance of the original image and $\sigma_{e}^{2}$ is the variance of error (Difference between the original and denoised image). Brighter regions have a stronger signal due to more light, resulting in higher overall SNR.

Peak Signal-to-Noise Ratio (PSNR): The PSNR represents the size of the error relative to the peak value of the signal than with the size of the error relative to the average squared value of the signal and is computed a with the size of the error relative to the average squared value of the signal and is computed as

$$
\text { PSNR }=10 \log _{10}\left(\frac{S^{2}}{\mathrm{MSE}}\right),
$$

where $S$ is the maximum intensity in the original image. The PSNR is higher for a better-transformed image and lower for a poorly transformed image. It measures image fidelity, that is, how closely the transformed image resembles the original image.

Correlation Coefficient (CC): It represents the strength and direction of a linear relationship between two variates. The best known is the Pearson product moment correlation coefficient, which is obtained by dividing the covariance of the two variables by the product of their standard deviation, as given by

$$
\mathrm{CC}=\frac{N \sum \mathrm{X}_{i} \mathrm{Y}_{i}-\sum \mathrm{X}_{i} \sum \mathrm{Y}_{i}}{\sqrt{N \sum \mathrm{X}_{i}^{2}-\left(\sum \mathrm{X}_{i}\right)^{2}} \sqrt{N \sum \mathrm{Y}_{i}^{2}-\left(\mathrm{Y}_{i}\right)^{2}}} \cdot
$$

If the correlation coefficient is near to +1 , then there exists stronger positive correlation between the original and despeckled image

\section{EXPERIMENTATION USING DISCRE- TE WAVELET TRANSFORM, LAPLAC- IAN PYRAMID TRANSFORM AND CONTOURLET TRANSFORM}

The experimentation has been performed on both the medical ultrasound images and the synthetic images in order to ascertain the effectiveness of the despeckling methods.

\subsection{Medical ultrasound images}

The experimentation is carried out on 52 ultrasound images of size 512 X 512 of kidney using wavelet transform, Laplacian pyramid transform and contourlet transform based despeckling methods. These images are acquired using the instrument GE LOGIQ 3 Expert system with $5-\mathrm{MHz}$ transducer frequency, in JPEG format. The three methods have been implemented on the Core2Duo system with $1 \mathrm{~GB}$ RAM and $2.53 \mathrm{GHz}$ using MATLAB 7.9.

\subsubsection{Discrete wavelet transform}

In [4], the experimental results show that wavelet transforms can denoise the speckle images more effectively. The main advantage of the wavelet transform is that the image fidelity after reconstruction is visually lossless. Further, it is found that the wavelet filter bior 6.8 with level 3 yields better results than other filters. The performance evaluation of the wavelet method is done in terms of Variance, MSE, SNR, PSNR and CC values computed from the despeckled image. The average results obtained for 52 medical ultrasound images for the different thresholding schemes are given in the Table 1. From Table 1 it is observed that subband threshold function, using Bayes shrinkage rule and soft thresholding technique, gives superior results than other thresholding techniques. The results of wavelet method are compared with common speckle filters, namely the Kuan, Wiener and Lee filters (Figures 1-3). The comparison shows that the wavelet method is better than common speckle filters.

\subsubsection{Laplacian pyramid transform}

The Laplacian pyramid transform is performed on the log transformed image. The Laplacian pyramidal decompositions up to nine levels are performed using biorthogonal filters with 
sufficient accuracy numbers such as the " $9 / 7$ " and " $5 / 3$ ". Further, thresholding schemes such as hard thresholding, soft thresholding and semi-soft thresholding is performed to reduce speckle. The threshold value is calculated using Bayes'shrinkage rule. The Table 2 shows the average results obtained for 52 ultrasound images for different reconstruction methods. The PSNR is calculated up to nine LP decompositions. The PSNR value increases up to 5 decompositions using ST, 2 decompositions using SST and 1 decomposition using HT thereafter reduces. Hence, the optimal level of LP decomposition is 5 for soft thresholding (LP-ST-L5), 2 decompositions for semisoft thresholding (LP-SST-L2) and 1 decomposition for hard thresh olding (LP-HT-L1). The optimal reconstruction method is determined by criteria, namely lower variance and MSE, higher SNR and PSNR values, Correlation Coefficient (CC) is nearly equal to one. From Table 2, it is observed that the one level Laplacian pyramidal decomposition using hard thresholding and the filter bior $5 / 3$ yield better results than other techniques. The reconstructed image is the despeckled image.

\subsubsection{Contourlet transform}

The contourlet transform is performed using double filter bank structure. The six levels of Laplacian pyramidal decompositions are performed using biorthogonal filters with sufficient accuracy numbers such as the "9-7". The directional decompositions up to nine is performed in all the pyramidal levels, using two dimensional ladder filters. The contourlet transform uses the "9/7" filters in LP stage because, in the multiscale decomposition stage, it significantly reduces all inter-scale, inter-location and inter direction mutual information of contourlet coefficients. Similarly, in directional decomposition stage, the ladder structure PKVA filters are more effective in localizing edge direction as these filters reduce the interdirection mutual information. Further, thresholding schemes such as hard thresholding, soft thresholding or semi-soft thresholding is performed to reduce speckle. The threshold value is calculated using Bayes' shrinkage rule. The PSNR is calculated up to 6 LP decompositions. The PSNR value increases up to 2 decompositions using HT, ST and SST, and thereafter reduces. Hence, the optimal level of LP decomposition is 2 . The average results obtained for 52 ultrasound images for different reconstruction methods are tabulated. The results obtained for the optimal decomposition of LP levels and directional decompositions using contourlet method based on hard thresholding, soft thresholding and semisoft thresholding using Bayes rule are given in Tables 3, 4 and 5, respectively. The optimal reconstruction method is determined by the criteria, namely, lower Variance and MSE, higher SNR and PSNR values, correlation coefficient is nearly equal to one. From the Tables 3, 4 and 5, it is observed that the 2-level Laplacian pyramidal decomposition and 6 directional bandpass sub-bands (2 at level 1, 4 at level 2) using hard thresholding yield better results than soft thresholding and semi-soft thresholding techniques. The frequency bands obtained by using optimal level L2-21 of contourlet decomposition are as follows: the $2^{\text {nd }}$ level has 1 approximation band of size $64 \times 64$ and 6 detail components ( 4 of 64 x 64, 2 of $128 \times 256$ ). The reconstructed image is the despeckled image. The hard thresholding is better than other thresholding methods, because small coefficients are removed while others are left untouched in
Table 2. Performance evaluation of different thresholding methods in terms of filter assessment parameters

\begin{tabular}{|c|c|c|c|c|c|}
\hline \multicolumn{7}{|c|}{ Filter type $=$ Bior 5/3 } \\
\hline Levels & PSNR & SNR & MSE & Variance & CC \\
\hline LP-HT-L1 & $\mathbf{2 3 . 8 7 7}$ & $\mathbf{1 8 . 5 9 8}$ & $\mathbf{0 . 0 1 3 8}$ & $\mathbf{0 . 0 1 6 0}$ & $\mathbf{0 . 9 9 6}$ \\
\hline LP-ST-L5 & 20.181 & 16.792 & 0.0161 & 0.0179 & 0.986 \\
\hline LP-SST-L2 & 20.9825 & 17.069 & 0.0155 & 0.0169 & 0.993 \\
\hline \multicolumn{7}{|c|}{ Filter type $=$ Bior 9/7 } \\
\hline LP-HT-L1 & 22.495 & 16.246 & 0.019 & 0.016 & 0.996 \\
\hline LP-ST-L5 & 21.328 & 15.570 & 0.016 & 0.016 & 0.987 \\
\hline LP-SST-L2 & 20.832 & 15.964 & 0.022 & 0.020 & 0.992 \\
\hline
\end{tabular}

HT while in ST or SST, coefficients above the threshold are shrunk by absolute value of threshold.

The filter assessment parameters MSE, SNR, PSNR, variance and $\mathrm{CC}$ are computed for all the three transforms. The Figures 46 , shows the comparison of the three methods. From the Figures 4-6, it is observed that, the contourlet based despeckling method shows better performance than the other despeckling methods. Further, it is found that the despeckling using contourlet transform gives better results than the speckle reduction method based on wavelet transform in particular. The wavelet based Bayes shrink thresholding method is based on separable 2D wavelet transform has limited directions (Horizontal, Vertical and Directional). Speckle noise in medical ultrasound images will generate significant coefficients in wavelet domain just like true detail features, such as edges. The despeckling method in contourlet domain can combine the coefficients along the smooth curve, like the cyst edges and kidney stone contour. However, the speckle noise is less likely to generate significant coefficients using the contourlet method, and thus, it directly leads to better performance in suppressing noise than the Bayes shrink thresholding scheme based on wavelet domain. The medical experts performed the visual inspection to confirm the improvement of the image quality attained by the CT algorithm in our experiment.

Another way to analyze the effect of filters is to study the error image between the original image and the filtered image. The error image is calculated as the absolute difference between the two images. The distribution of information inside the error image provides a qualitative indication about the filtering method. If the information is randomly distributed, generally similar to speckle distribution, this indicates that the extracted information from original image is non-structured and may be considered to be mainly noise. If there is some structured information is present in the error image, this indicates that some structured information (boundaries or edges) is higher in one image than other. The difference between the original image and the filtered image proves that edges in the original are higher in amplitude than the filtered image this indicates that a smoothing of edges was obtained. If the opposite case happens, the indication is that a boosting of the edges is present. The error image concerning contourlet transform in the Figure 10 (f) shows clearly that a random distribution of information is present in this figure, which is very similar to speckle distribution. This error image presents a map distribution 
Table 3. Optimal decomposition of LP levels and directional decompositions in terms of variance, MSE, SNR, PSNR, CC values using contourlet method based on hard thresholding (HT) using Bayes rule.

\begin{tabular}{|c|c|c|c|c|c|}
\hline Levels & PSNR & Variance & MSE & CC & SNR \\
\hline L1-2 & 32.492 & 0.00881 & 0.00064 & 0.9993 & 26.743 \\
\hline L2-21 & $\mathbf{3 2 . 7 7 0}$ & $\mathbf{0 . 0 0 8 1}$ & $\mathbf{0 . 0 0 0 6 0}$ & $\mathbf{0 . 9 9 9 5}$ & $\mathbf{2 7 . 1 7 7}$ \\
\hline L3-521 & 26.704 & 0.00882 & 0.00303 & 0.9977 & 25.126 \\
\hline $\begin{array}{c}\text { L4- } \\
\text { 4311 }\end{array}$ & 25.391 & 0.00872 & 0.00463 & 0.9973 & 24.853 \\
\hline $\begin{array}{c}\text { L5- } \\
\text { 51311 }\end{array}$ & 25.325 & 0.00882 & 0.00470 & 0.9977 & 23.823 \\
\hline $\begin{array}{c}\text { L6- } \\
121311\end{array}$ & 25.312 & 0.00982 & 0.00470 & 0.9977 & 21.811 \\
\hline
\end{tabular}

Table 4. Optimal decomposition of LP levels and directional decompositions in terms of variance, MSE, SNR, PSNR, CC values using contourlet method based on soft thresholding (ST) using Bayes rule.

\begin{tabular}{|c|c|c|c|c|c|}
\hline Levels & PSNR & Variance & MSE & CC & SNR \\
\hline L1-1 & 28.394 & 0.00962 & 0.00154 & 0.99910 & 21.845 \\
\hline L2-21 & 29.153 & 0.00923 & 0.00134 & 0.99853 & 24.474 \\
\hline L3-332 & 20.284 & 0.00934 & 0.01332 & 0.99358 & 20.862 \\
\hline $\begin{array}{c}\text { L4- } \\
5331\end{array}$ & 22.837 & 0.00905 & 0.00863 & 0.99288 & 19.115 \\
\hline $\begin{array}{c}\text { L5- } \\
\text { 22332 }\end{array}$ & 21.620 & 0.00947 & 0.01077 & 0.99292 & 18.058 \\
\hline $\begin{array}{c}\text { L6- } \\
123332\end{array}$ & 22.041 & 0.00947 & 0.01033 & 0.99271 & 19.420 \\
\hline
\end{tabular}

Table 5. Optimal decomposition of LP levels and directional decompositions in terms of variance, MSE, SNR, PSNR, CC values using contourlet method based on semi-soft thresholding (SST) using Bayes rule.

\begin{tabular}{|c|c|c|c|c|c|}
\hline Levels & PSNR & Variance & MSE & CC & SNR \\
\hline L1-1 & 30.468 & 0.00963 & 0.00155 & 0.99911 & 22.962 \\
\hline L2-21 & 30.718 & 0.00923 & 0.00134 & 0.99852 & 25.269 \\
\hline L3-332 & 24.575 & 0.00935 & 0.01332 & 0.99358 & 23.074 \\
\hline $\begin{array}{c}\text { L4- } \\
5331\end{array}$ & 24.558 & 0.00906 & 0.00864 & 0.99288 & 22.997 \\
\hline $\begin{array}{c}\text { L5- } \\
\text { 22332 }\end{array}$ & 24.189 & 0.00947 & 0.01077 & 0.99292 & 20.987 \\
\hline $\begin{array}{c}\text { L6- } \\
123332\end{array}$ & 24.200 & 0.00948 & 0.01034 & 0.99272 & 21.694 \\
\hline
\end{tabular}

on every pixel in the image which is much better representation than mean square error or other, generally calculated on the whole image, which is of quite small interest in this type of highly noisy data. This type of error representation proves visually that the edges neither amplified nor damped and the random distribution of noise shows that the filtering reduces mainly the speckle. The error image in the Figure 10 (c) and Figure 10(d) concerning the wavelet transform and Laplacian pyramid transform respectively, shows that some structures(edges) and non-structures (mainly noise) information are amplified in these images.Figure 10(d) concerning the wavelet transform and Laplacian pyramid transform respectively, shows that some structures(edges) and nonstructures (mainly noise) information are amplified in these images. This is due to the boosting of local strong variation without taking into account the directionality as in contourlet. The qualitative assessment and quantitative viewpoints shows that despeckling using contourlet transform is better adapted to ultrasound images.

\subsection{Synthetic image}

It is very difficult to compare three methods for removing speckle from real ultrasound images, because it depends on what we need from such an image. For small details it is not obvious to a non-expert in the diagnoses of ultrasound images to know what we need to eliminate or to preserve and enhance. Some of the statistical measurements such as SNR, MSE, CC are not very significant in the case of real ultrasound images if they are applied to whole image without a prior knowledge of it. Such measurements could be efficient in the case of simulated images. In order to investigate the quantitative performance of the selected methods, an image with artificial speckle noise was employed. A purely synthetic image which consists of regions with uniform intensity, sharp edges and strong scatters with SNR of $8.3 \mathrm{~dB}$ is considered for experimentation(image available at http://telin.ugnet.be/ sanja). The experimentation is carried out on synthetic image to evaluate despeckle filtering in medical ultrasound imaging. All the three despeckling methods, namely, wavelet transform, Laplacian pyramid transform and contourlet transform are performed on the phantom image. All the filters show the similar performance in removing the speckle at the same time gave significant results. From the Figures.7-9, it is observed that despeckling using contourlet transform gave very good performance in terms of (i) decreasing the variance, (ii) increasing the SNR, PSNR and CC values to be nearly equal to one. Further, it is found that, 4-level of Laplacian pyramidal decomposition and 14 directional bandpass subbands ( 4 of $32 \times 32,4$ of 64 x 64,2 of $128 \times 256,4$ of 256X256) using soft thresholding with Bayes shrinkage rule yields optimal results for speckle reduction. From Figure 11 (a), (c), (e), the edges of the phantom image was studied and it was shown that the contourlet transform based despeckling method does not blur edges as with the other two despeckle filtering techniques. The error image in Figure 11 (b) and (d), obtained after wavelet transform and Laplacian pyramid transform shows that structured information and nonstructured information are amplified (white pixels) in these images. This is due to the boosting of local strong variation without taking into account the edge direction as in contourlets (Figure 11 (f)). It is clear that despeckling using contourlet transform is better interms of smoothing the internal variations and preserving boundaries.

\section{CONCLUSION}

In this paper, three multi-scale methods are tested and compared for despeckling medical ultrasound images. These methods are proven to be very efficient to treat multiplicative noise and reduce speckle in ultrasound images. Several studies prove that wavelet based despeckling is among the best methods for ultrasound images. The Laplacian pyramid transform and contourlet transform based despeckling methods are applied recently on ultrasound images and proves to give very good results. But the wavelet transform, Laplacian pyramid transform and contourlet transform have not been compared to each other using ultrasound images, where lot of variations exists in the background. The performance evaluation of the three methods is done interms of variance, MSE, SNR, PSNR correlation coefficient (CC) are computed from despeckled image. This study proves that contourlet transform using hard thresholding is an excellent tool for despeckling medical ultrasound images. Further, it is found that, 2-level of Laplacian pyramidal decomposition and 6 directional bandpass subbands ( 4 of $64 \mathrm{x}$ 64,2 of $128 \times 256$ ) using hard thresholding with Bayes' shrinkage rule yields optimal results for speckle reduction. The 
error distribution indicates that the contourlet scheme provides a better overall improvement. The contourlet transform based despeckling method produces better quality ultrasound images for subsequent computer-assisted image analysis by medical experts.

\section{ACKNOWLEDGMENTS}

Authors are grateful to the reviewers for their helpful comments which improved the quality of the paper. Further, authors are thankful to Dr. Ramesh Mankare, Radiologist, Sangameshwar Scanning Centre, Bijapur, Karnataka, for providing the ultrasound images of kidney, and also for helpful discussions.

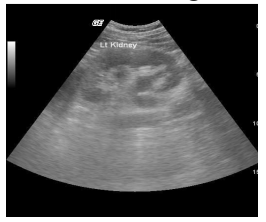

(a)

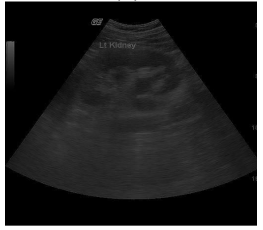

(d)

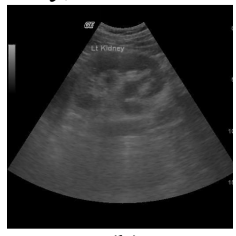

(b)

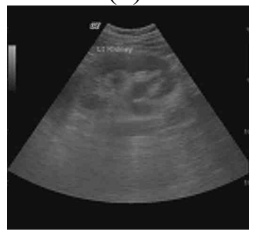

(e)

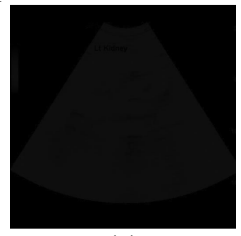

(c)

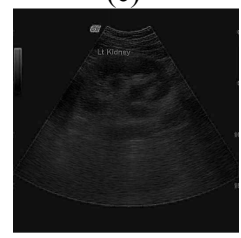

(f)
Fig.10 (a) Despeckled image using wavelet transform (b) Despeckled image using Laplacian pyramid transform (c)The difference image of original image and Fig. 10(a). (d) The difference image of original image and Fig. 10(b). (e) Despeckled image using contourlet transform (f) The difference image of original image and Fig.10 (e)

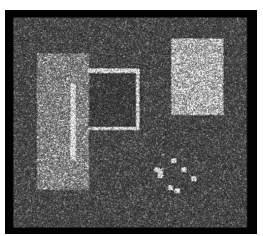

(a)

(d)

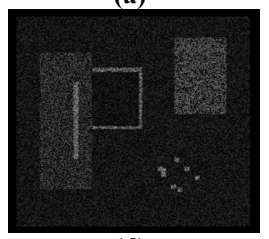

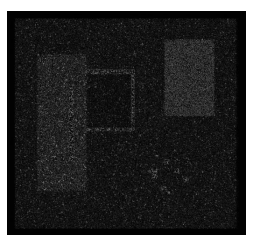

(b)

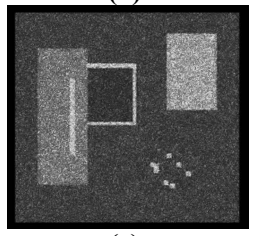

(e)

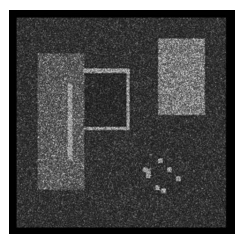

(c)

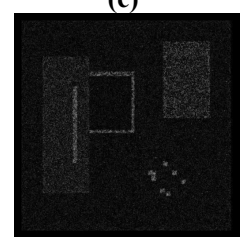

(f)
Fig. 11. (a) Despeckled image using wavelet transform (b) The difference image of original image and Fig. 11 (a). (c) Despeckled image using Laplacian pyramid transform (d) The difference image of original image and Fig. 11 (c). (e) Despeckled image using contourlet transform (f) The difference image of original image and Fig.11 (e)

\section{REFERENCES}

[1] Paul Suetens,2002,"Fundamentals of Medical Imaging", $1^{\text {st }}$ Edition, Cambridge University, U.K., pp.145-182.

[2] S. Kalaivani Narayanan and R.S.D.Wahidabanu 2009, A View of Despeckling in Ultrasound Imaging. Signal Processing, Image processing and Pattern Recognition (3),pp.85-98.
[3] N.K.Ragesh, A.R.Anil and R.Rajesh, 2011, Digital Image Denoising in Medical Ultrasound images: A Survey, ICGST AIML-11 Conference, Dubai, UAE, 12-14 April 2011,pp.67-73.

[4] P.S.Hiremath, Prema T.Akkasaligar, Sharan Badiger, 2010, Visual Enhancement of Digital Ultrasound Images using Multiscale Wavelet Domain, Pattern Recognition and Image Analysis, 20(3),pp. 303-315.

[5] Alin Achim, Anastasios Bezerianos, Panagiotis Tsakalides, 2001 Novel Bayesian Multiscale Method for Speckle Removal in Medical Ultrasound Images IEEE Transactions on Medical Imaging, 20,pp.772-783.

[6] A. Pizurica, W.Philips, Marc Acheroy, 2003 , A Versatile Wavelet Domain Noise Filtration Technique for Medical Imaging. IEEE Transactions on Medical Imaging, 22, pp.323-331.

[7] Nikhil gupta and M.N.Swamy,2005, Despeckling of Medical Ultrasound Images Using Data and Rate Adaptive Lossy Compression,IEEE Trans.Medical Imaging, vol.24, No.6, pp.682-695.

[8] Minh N. Do, Martin Vetterli.2003, Contourlets, In: Beyond Wavelets, G.V.Well, J Stoeckerand, (eds) Academic Press, pp.1-27.

[9] Mao-yu-Huang, yueh-Min Huang and Ming-Shi Wang ,2004,Speckle reduction of ultrasound image based on contourlet transform, Int. Computer Symposium, Dec. 1517, 2004, Taipei, Taiwan. pp.178-182.

[10] P.S.Hiremath, Prema T. Akkasaligar, Sharan Badiger. 2009, Despeckling Medical Ultrasound Images Using the Contourlet Transform. In: Proceedings of the $4^{\text {th }}$ AMS Indian International Conference on Artificial Intelligence (IICAI) ; pp.1814-27.

[11] K.Thangavel, R.Manavalan andI.Laurence, 2009, A.Removal of Speckle Noise from Ultrasoun Medical Image based on Special Filters: Comparative study, ICGST GVIP Journal ISSN1687-398x, vol.9, issue 3, pp.25-32.

[12] Wan Mahani Hafizah and Eko Supriyanto,2011, Comparative Evaluation of Ultrasound Kidney Image Enhancement Techniques, International Journal of Computer Applications (0975 - 8887) vol.21, No.7, pp.15-19.

[13] Mariana C.N., Luminita M. and Laura O, 2010, Comparative Approach for Speckle Reduction in Medical Ultrasound Images", Romanian J. Biophysics, Vol. 20, No.1 pp.13-21.

[14] Ali Saad, 2008 Simultenous Speckle Reduction and Constrast Enhancement for Ultrasound Images: Wavelet Versus Laplacian Pyramid", Int.Jou. of Pattern Recognition and Image Analysis, vol.18, No.1, pp.63-70.

[15] P. J. Burt and E. H. Adelson,1983, The Laplacian Pyramid as a compact image code, IEEE Trans.on Commun., vol. 31, No. 4, pp. 532-540.

[16] Minh N. Do, Martin Vetterli. 2005, The contourlet transform: Efficient directional Multiresolution Image Representation. IEEE Transactions on Image Processing 14 ,pp.2091-2106. 
Table 1. Performance evaluation of different thresholding methods in terms of variance, MSE, SNR, PSNR, CC values.

\begin{tabular}{|c|c|c|c|c|c|c|c|c|c|c|c|}
\hline $\begin{array}{c}\text { Shrinkage } \\
\text { rule }\end{array}$ & & \multicolumn{5}{|c|}{ Global thresholding(2) } & \multicolumn{5}{|c|}{ Subband thresholding(1) } \\
\hline \multirow{5}{*}{$\begin{array}{c}\text { Bayes } \\
\text { shrinkage } \\
\text { (2) }\end{array}$} & Thresholding & variance & MSE & SNR & $\mathrm{CC}$ & PSNR & variance & MSE & SNR & $\mathrm{CC}$ & PSNR \\
\hline & Hard(1) & 0.0552 & 0.00137 & 23.95 & 0.99613 & 26.6934 & 0.0237 & 0.0014 & 24.8 & 0.998 & 27.513 \\
\hline & Soft(2) & 0.0552 & 0.00137 & 23.95 & 0.99613 & 26.6934 & 0.0138 & 0.0012 & 24.811 & 0.999 & 28.66 \\
\hline & Semi-soft(3) & 0.0552 & 0.00139 & 23.98 & 0.99613 & $25 . .93$ & 0.0268 & 0.0014 & 24.78 & 0.998 & 27.722 \\
\hline & & \multicolumn{5}{|c|}{ Global thresholding } & \multicolumn{5}{|c|}{ Subband thresholding } \\
\hline \multirow{3}{*}{$\begin{array}{c}\text { universal } \\
\text { shrinkage } \\
\text { (1) }\end{array}$} & $\operatorname{Hard}(1)$ & 0.0248 & 0.0057 & 18.28 & 0.9933 & 21.3518 & 0.0288 & 0.0056 & 20.28 & 0.996 & 20.827 \\
\hline & Soft(2) & 0.0142 & 0.0164 & 13.83 & 0.9875 & 17.7381 & 0.0214 & 0.0085 & 16.74 & 0.994 & 18.198 \\
\hline & Semi-soft(3) & 0.0198 & 0.0094 & 16.04 & 0.9919 & 17.0855 & 0.0262 & 0.0073 & 18.984 & 0.995 & 19.612 \\
\hline
\end{tabular}

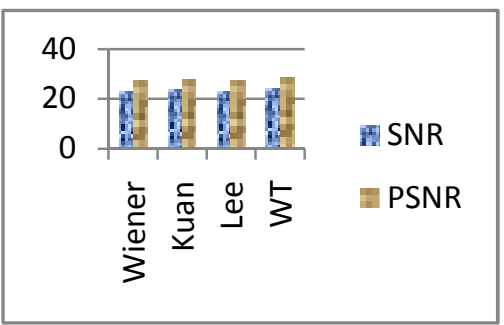

Fig. 1. Statistical features PSNR and SNR for common speckle filtersand Wavelet filter.

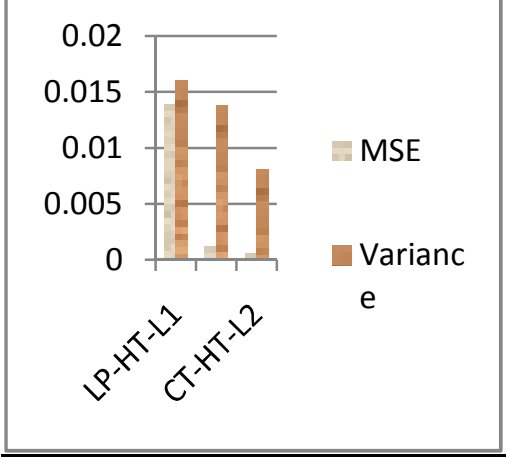

Fig. 4. Statistical feature MSE, Variance for CT , LP and WT for US images.

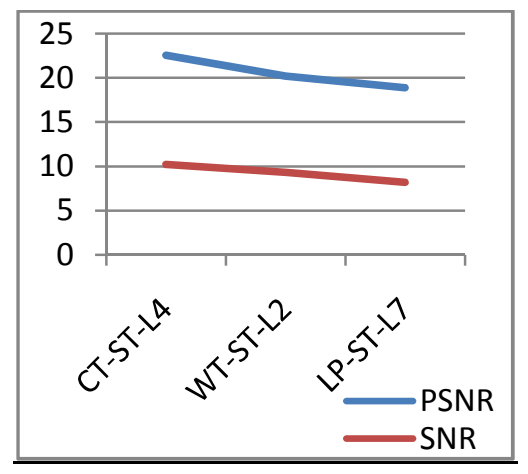

Fig. 7. Statistical feature PSNR, SNR for CT, LP and WT for Synthetic image.

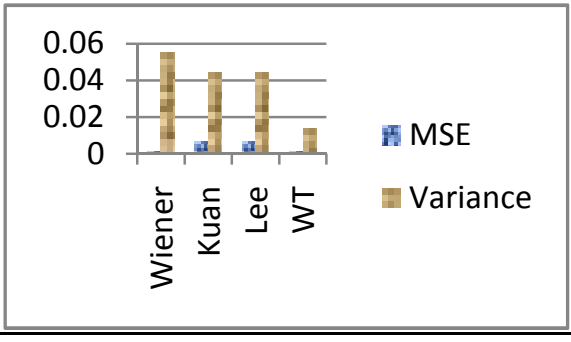

Fig. 2. Statistical features Variance, MSE for common speckle filters and Wavelet filter.

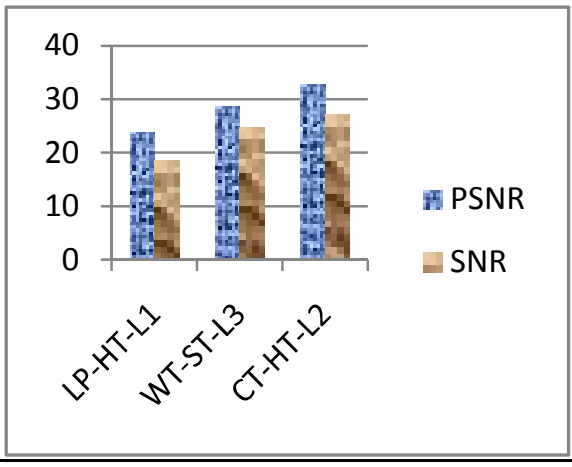

Fig. 5. Statistical feature PSNR, SNR for CT, LP and WT for US images.

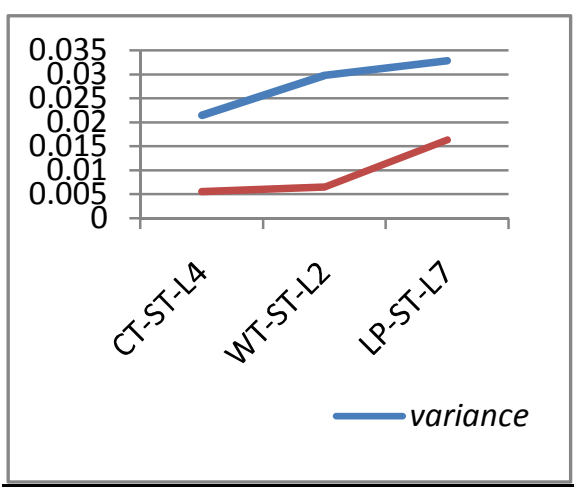

Fig. 8. Statistical feature MSE, Variance for CT, LP and WT for Synthetic image.

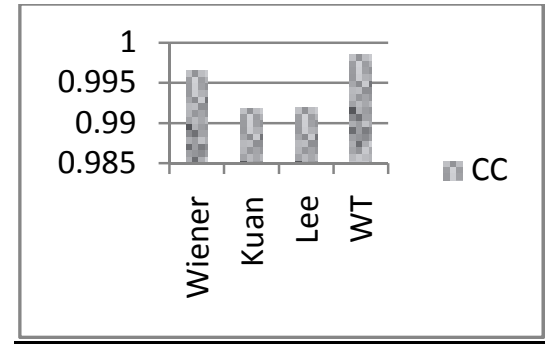

Fig. 3. Statistical feature Correlation Coefficient (CC)for common Speckle filters and Wavelet.

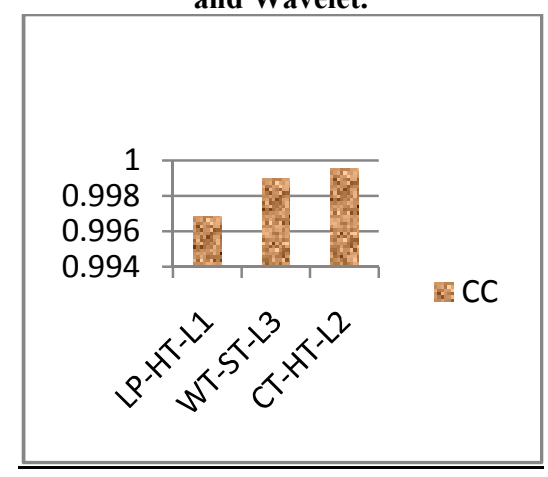

Fig. 6. Statistical feature Correlation Coefficient (CC), for CT, LP and WT for US images.

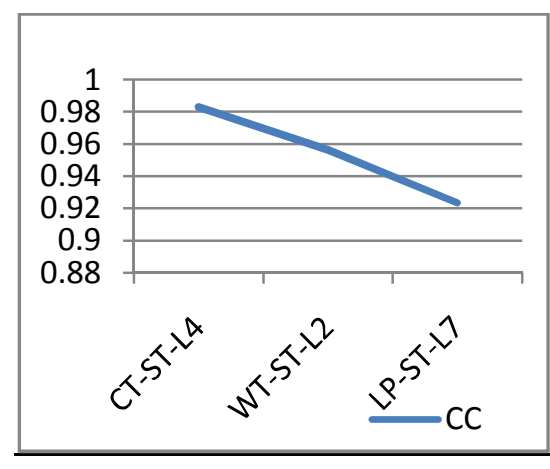

Fig. 9. Statistical feature Correlation Coefficient (CC), for CT, LP and WT for Synthetic image. 